\title{
Stochastic Polarization Switching Dynamics in Vertical-Cavity Surface-Emitting Lasers: Theory and Experiment
}

\author{
Jan Danckaert, Member, IEEE, Michael Peeters, Member, IEEE, Claudio Mirasso, Maxi San Miguel, \\ Guy Verschaffelt, Associate Member, IEEE, Jan Albert, Bob Nagler, Heiko Unold, Rainer Michalzik, \\ Giovanni Giacomelli, and Francesco Marin
}

\begin{abstract}
We present an analytical, numerical, and experimental study of the switching time and jitter of current-induced polarization switching in vertical-cavity surface-emitting lasers (VCSELs) as an example of switching in a nonlinear system in the presence of noise. Assuming that the switching is induced by changes in the dichroism, the problem can be reduced to the first-passage-time problem in gain-switched Class-A lasers. The theoretical results show excellent agreement both with numerical simulations based on the full-rate equations model and with experiments performed on oxide-confined VCSELs.
\end{abstract}

Index Terms-Noise, polarization effects, semiconductor lasers, switching, vertical-cavity surface-emitting lasers (VCSELs).

\section{INTRODUCTION}

A LTHOUGH vertical-cavity surface-emitting lasers (VCSELs) are nowadays employed in a wide variety of applications, not all their properties are completely understood.

Manuscript received January 9, 2004; revised May 26, 2004. This work was supported in part by the Belgian government under the Interuniversity Attraction Pole program (IAP V/18), in part by the Concerted Research Action, and in part by the Research Council of the Vrije Universiteit Brussel. The collaboration between the groups in Brussels, Palma de Mallorca, Ulm, and Florence was made possible through the European RTN network VISTA under Contract HPRN-CT-2000-00034. Additional support from, and discussions within, the framework of the European COST actions 268 and 288 are acknowledged. The work of J. Danckaert, G. Verschaffelt, and B. Nagler was supported by the Fund for Scientific Research-Flanders.

J. Danckaert is with the Department of Applied Physics and Photonics, Vrije Universiteit Brussel, B-1050 Brussels, Belgium, and also with IMEDEA, Campus Universitat de les Illes Balears, E-07122 Palma de Mallorca, Spain (e-mail: jandan@vub.ac.be).

M. Peeters, G. Verschaffelt, and J. Albert are with the Department of Applied Physics and Photonics, Vrije Universiteit Brussel, B-1050 Brussels, Belgium.

B. Nagler was with the Department of Applied Physics and Photonics, Vrije Universiteit Brussel, B-1050 Brussels, Belgium. He is now with the Lawrence Berkeley National Laboratory, Berkeley, CA 94720 USA.

C. Mirasso is with Departament de Fisica, Universitat de les Illes Balears, E-07122 Palma de Mallorca, Spain.

M. San Miguel is with IMEDEA (UIB-ESIC), Campus Universitat de les Illes Balears, E-07122 Palma de Mallorca, Spain.

H. Unold was with the Optoelectronics Department, University of Ulm, D-89069 Ulm, Germany. He is now with the Institute of Quantum Electronics, Swiss Federal Institute of Technology, CH-8093 Zurich, Switzerland.

R. Michalzik is with the Optoelectronics Department, University of Ulm, D-89069 Ulm, Germany.

G. Giacomelli is with Istituto Nazionale di Ottica Applicata and National Institute for the Physics of Matter, 50125 Firenze, Italy.

F. Marin is with the Department of Physics, Universita Firenze, National Institute for the Physics of Matter, and the European Laboratory for Nonlinear Spectroscropy, 50019 Sesto F.no (FI), Italy.

Digital Object Identifier 10.1109/JSTQE.2004.837014
Polarization switching (PS) between the two orthogonal polarization modes (PMs) when the injected current is changed has been experimentally observed and studied for a decade now [1]-[4]. While uncontrolled PS is highly undesirable in many applications, controlled current-induced PS might be interesting as an alternative switching mechanism [5], [6]. However, the dynamics of current driven PS in VCSELs is still unclear. In this paper, we investigate the PS dynamics in VCSELs as an example of switching in a nonlinear system in the presence of noise. Previous experimental studies have demonstrated that stochastic effects are indeed important in PS, and manifest themselves in, for example, spontaneous mode hopping [7], [8] or anomalously large jitter in modulation experiments [9]. Besides, our study also reveals some information about the origin of PS in our oxide-confined VCSELs. The different mechanisms proposed to explain PS in VCSELs can roughly be divided into two categories: those invoking slow (lattice) thermal mechanisms [1], [4], [10] and those relying on faster mechanisms [2], [11]-[15]. PS of both thermal and nonthermal origin has been demonstrated experimentally [9], [16] by measuring the polarization modulation frequency response. Here, we study the step response of PS of nonthermal origin in the time domain and derive analytical expressions for the switching time and its variance, and compare these results with experiments on an oxide confined VCSEL.

\section{Model AND Stochastic TheORY}

The model we are using is a phenomenological one, where PS is supposed to be caused by changes in the gain balance (or dichroism) between the two PMs. The disadvantage of using such an ad-hoc model is of course that the mechanism(s) underlying the polarization selection remain somewhat hidden, although at the end of this paper we will draw some conclusion with respect to this mechanism(s). The main advantage of using such an ad-hoc model is that it can be used whatever the mechanism(s) causing the changes in dichroism. We start from the following stochastic rate equations:

$$
\begin{aligned}
\frac{d E_{x, y}^{\prime}}{d t} & =\frac{1+i \alpha}{2}\left(G_{x, y}-\tau_{x, y}^{-1}\right) E_{x, y}^{\prime}+\sqrt{\beta^{\prime} N} \xi_{x, y}(t) \\
\frac{d N}{d t} & =\frac{I}{e}-\frac{N}{\tau_{c}}-G_{x} P_{x}-G_{y} P_{y} .
\end{aligned}
$$


Here, $E_{x, y}^{\prime}$ are the slowly varying amplitudes of the $x(y)$ polarized electric fields, $N$ is the carrier number, $I$ is the injected current, $\tau_{x, y}$ are the photon life times, while $\tau_{c}$ is the carrier relaxation time. The gain due to stimulated emission in each of the modes is described by $G_{x, y}=G_{x, y}\left(N, P_{x}, P_{y}, I, T, \ldots\right)$ that are usually complicated functions of the variables $N$ and $P_{x, y}=\left|E_{x, y}^{\prime}\right|^{2}$, and that moreover depend on some of the parameters such as the current $I$ and/or the temperature $T$. Henry's $\alpha$-factor describes phase-amplitude coupling in semiconductor lasers, but plays no further role here. The factor $\beta$ ' describes the strength of the spontaneous emission noise. The spontaneous emission noise is modeled through Langevin force terms $\xi_{i}(t)$, that are zero-mean, delta-correlated, complex Gaussian white noise terms

$$
\left\langle\xi_{k}(t) \xi_{l}^{*}\left(t^{\prime}\right)\right\rangle=\delta_{k, l} \delta\left(t-t^{\prime}\right)
$$

Noise contributions originating from the carriers are neglected. We now assume that the gain medium can be described adequately by a gain linear in $N$, but self- and cross-mode saturating with increasing optical power (coefficients $e_{s}$ and $e_{c}$, respectively)

$$
G_{x, y}=g_{x, y}\left(N-N_{\mathrm{tr}}\right)\left(1-e_{s} P_{x, y}-e_{c} P_{y, x}\right)
$$

where $N_{\mathrm{tr}}$ is the transparency carrier number and $g_{x, y}$ the gain coefficients. Equations (1) and (2) are typical rate equations for a standard two-mode semiconductor laser. In the specific case of a VCSEL, they can be deduced from the spin flip model [17]. This reduction [18] is valid for relatively large birefringence and spin relaxation rate. The spin flips are then essentially contributing to the cross-mode saturation terms $e_{c}$. The effects of self-saturation in the framework of the spin flip model have also been considered [19]. Very recently, it was shown that the polarization-dependent saturation of the intersubband absorption also contributes to an effective cross-mode saturation that is larger than the self-saturation [20]. This is important here as we will see that it is the diffrence between the self- and the cross-saturation that will play a major role in the switching dynamics.

Equations (1)-(2) can be rewritten taking advantage of the almost perfect degeneracy of the two polarization modes [21]. We introduce the dimensionless field amplitudes $E_{x, y}$ and intensities $p_{x, y}=\left|E_{x, y}\right|^{2}$, the deviation $\eta=\rho^{-1}\left[g\left(N / N_{\text {tr }}-1\right)-1\right]$ of the carrier density from the clamped value, as well as the relative linear dichroism between the two modes $G=\rho^{-1}\left(g_{y}-g_{x}\right) / g_{x}$ and the current reduced to threshold $J=\left(I-I_{\mathrm{th}}\right) / I_{\mathrm{th}}$, yielding

$$
\begin{aligned}
\dot{E}_{x}= & \frac{1}{2}(1+i \alpha)\left(\eta-\epsilon_{s} p_{x}-\epsilon_{c} p_{y}\right) E_{x} \\
& +\sqrt{\beta} \xi_{x}(t) \\
\dot{E}_{y}= & \frac{1}{2}(1+i \alpha)\left(\eta+G-\epsilon_{s} p_{y}-\epsilon_{c} p_{x}\right) E_{y} \\
& +\sqrt{\beta} \xi_{y}(t) \\
\dot{\eta}= & \frac{1}{\rho}\left(J-p_{x}-p_{y}\right)-\eta-\left(\eta-\epsilon_{s} p_{x}-\epsilon_{c} p_{y}\right) p_{x} \\
& -\left(\eta+G-\epsilon_{s} p_{y}-\epsilon_{c} p_{x}\right) p_{y} .
\end{aligned}
$$

Here, time has been reduced to the carrier lifetime and $\rho$ is the ratio of the photon and the carrier lifetimes: $\rho=\tau_{x} / \tau_{c}=10^{-3}$.
The field amplitudes and the current have been reduced in such a way that the lasing solutions are $p_{x}=J$ and $p_{y}=(J+\rho G)$, respectively (neglecting gain saturation and spontaneous emission noise, see [21] for the details). Also, the gain saturation parameters have been rescaled: $e_{s, c} \propto \rho \epsilon_{s, c}$. A stability analysis of (5)-(7) reveals that polarization switching can happen for certain parameter values [21]. We assume that the linear dichroism $G$ varies with current and changes sign. Then, polarization switching occurs at the current where $G=0$, the saturation parameters defining a bistable region around this point. Polarization switching is also possible for a fixed value of the linear dichroism $G$, but then the saturation coefficients need to be taken asymmetric (i.e., $\epsilon_{x y} \neq \epsilon_{y x}$ ) [18]. The theory developed below can also be applied to this case. A straightforward numerical simulation of a gain-induced switching event shows the carrier density to be essentially clamped during a switch. This observation points to a further simplification that can also be understood mathematically as follows. The first term on the right-hand side of (7) is dominating, so

$$
p_{x}+p_{y}=J+O(\rho) .
$$

This conservation law physically states that-on time scales longer than the inverse of the relaxation oscillation frequency - the two optical modes are anticorrelated. Several authors have indeed reported anticorrelation at low frequencies between the two polarization modes in a VCSEL [22]-[25]. The problem can now be further reduced into a single nonlinear dynamical equation [8], [21]. If, for example, $E_{x}$ is lasing $\left(p_{x}=J\right.$ and $\left.p_{y} \ll J\right)$, a switch-on of the $y$ mode will occur as the current is changed across the switching point. Then, (5) follows $\eta=\epsilon_{s} J$, and henceforth

$$
\dot{E}_{y}=\frac{1+i \alpha}{2}\left[(G-\Delta)+A\left|E_{y}\right|^{2}-B\left|E_{y}\right|^{4}\right] E_{y}+\sqrt{\beta} \xi_{y}(t)
$$

with the nonlinear dichroism $\Delta=\left(\epsilon_{c}-\epsilon_{s}\right) J$ being a remnant of the gain/loss nonlinearities. The coefficients of the nonlinear terms $A$ and $B$ are given elsewhere [8], but do not matter for the present purposes. The equivalent equation for the $x$ mode is obtained by changing the sign of $G$. The sufficient condition to have a (small) region of bistability around the switching current defined by $G\left(J_{\mathrm{sw}}\right)=0$ is $\Delta>0$.

Equation (9) is a class-A laser equation, valid on a time scale larger than the inverse of the relaxation oscillation frequency. It may come as a surprise that a class-A model can be applied to VCSELs, that are, like all semiconductor lasers, class-B lasers. However, we can note that, above laser threshold, the carrier density is essentially clamped, so that the carrier dynamics can be eliminated from the model. In fact, the conservation law (8) is a direct consequence of the clamping of the carriers. Secondly, as already stated, our model is only valid on time scales longer than the inverse of the relaxation oscillation frequency. As we will see, PS in VCSELs is a relatively slow phenomenon, happening on a time scale where the relaxation oscillations have died down. We have already applied a reduced equation like (9) to analyze the polarization mode hopping in VCSELs at constant current, with good agreement between theory and experi- 
ment [8]. There, we also verified the reduction numerically: the deviations between predictions for the average residence time from the reduced model [one-dimensional (1-D)] and the full model [three-dimensional (3-D)] never exceed 15\%.

Calculating the switching time $t^{*}$ (i.e., the time in which $p_{y}$ crosses a reference intensity $p_{0}$ ), is identical to the stochastic problem of the first passage time (or escape time) in class-A lasers [26], [27]. We briefly repeat the basic steps in the framework of the quasi-deterministic theory. In order for the nonlasing $y$ mode to ignite and depart from the noise level, we suppose that at $t=0$ a current step is applied such that $G-\Delta$ changes from an initial value $\left(G_{i}-\Delta_{i}\right)<0$ to a final value $\left(G_{f}-\Delta_{f}\right)>0$. We assume that the dichroism $G$ changes instantaneously with current. The linearized (9) (i.e., $A=B=0$ ) admits solutions

$$
E(t)=h(t) \exp \left[\frac{1+i \alpha}{2}\left(G_{f}-\Delta_{f}\right) t\right] .
$$

Here, $h(t)$ is a Gaussian stochastic function with zero mean, given by

$$
h(t)=\sqrt{\beta} \int_{0}^{t} \xi\left(t^{\prime}\right) \exp \left[-\frac{1+i \alpha}{2}\left(G_{f}-\Delta_{f}\right) t^{\prime}\right] d t^{\prime}+E(0) .
$$

Equation (10) can be modulus squared and inverted to solve for the passage time $t^{*}$, defined as the time in which a reference intensity value $p_{0}$ is crossed for the first time

$$
t^{*}=\frac{1}{G_{f}-\Delta_{f}} \ln \left(\frac{p_{0}}{|h(t)|^{2}}\right) .
$$

One can see that the Gaussian distribution $h(t)$ is acting as an effective initial condition. The passage time statistics depends on the covariance $\left\langle|h(t)|^{2}\right\rangle$. Assuming that $t \gg\left(G_{f}-\Delta_{f}\right)^{-1}$, this covariance can be approximated by

$$
\left\langle h^{2}\right\rangle=\left\langle|h(\infty)|^{2}\right\rangle=\frac{2 \beta}{\left(G_{f}-\Delta_{f}\right)}+\left|\frac{2 \beta}{\left(G_{i}-\Delta_{i}\right)}\right| .
$$

Here, $\left\langle|E(0)|^{2}\right\rangle$ has been replaced by the average value of the intensity below the switching point $\left|\left(2 \beta /\left(G_{i}-\Delta_{i}\right)\right)\right|$, i.e., the average intensity of the nonlasing mode. The passage time statistics can now be calculated by averaging $t^{*}$ over the Gaussian distribution

$$
P(h)=\frac{1}{\pi\left\langle h^{2}\right\rangle} \exp \left[-\frac{h^{2}}{\left\langle h^{2}\right\rangle}\right]
$$

The mean first passage time $\left\langle t^{*}\right\rangle$ and its variance $\left\langle\Delta t^{* 2}\right\rangle$ are then readily obtained

$$
\begin{aligned}
\left\langle t^{*}\right\rangle & =\frac{1}{\left(G_{f}-\Delta_{f}\right)}\left(\ln \left(\frac{p_{0}}{\left\langle h^{2}\right\rangle}\right)-\Psi(1)\right) \\
\left\langle\left(\Delta t^{*}\right)^{2}\right\rangle & =\frac{\Psi^{\prime}(1)}{\left(G_{f}-\Delta_{f}\right)^{2}}
\end{aligned}
$$

where $\Psi$ is the digamma function $\left(\Psi(1)=-0.577\right.$ and $\Psi^{\prime}(1)=$ 1.64).
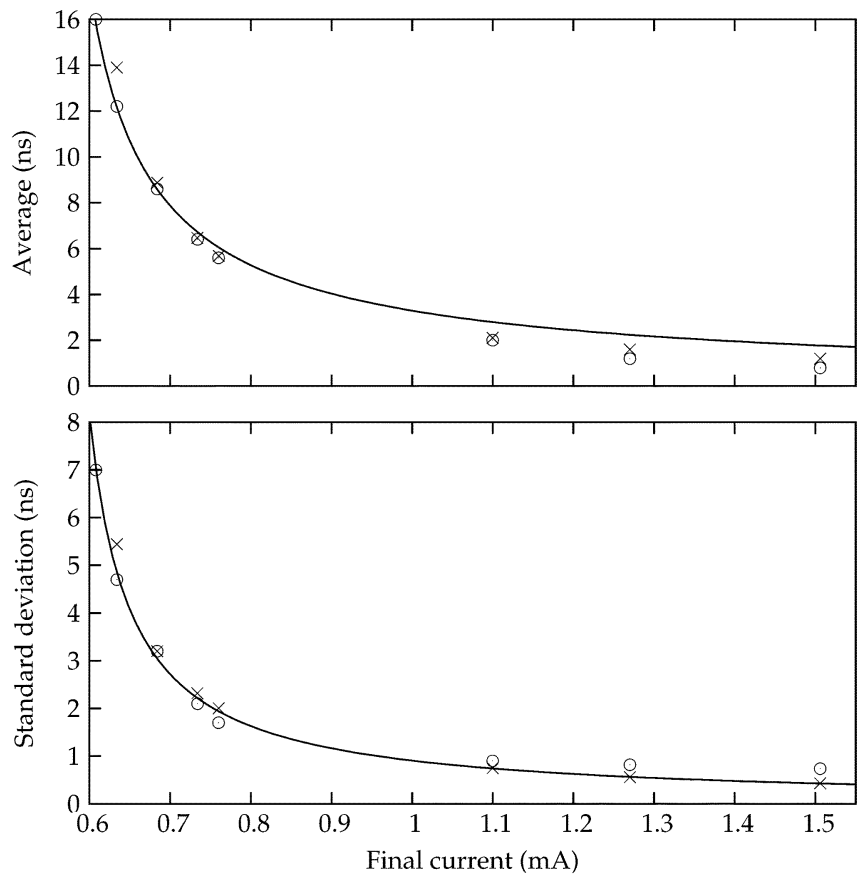

Fig. 1. Average switching time $\left\langle t^{*}\right\rangle$ (upper figure) and its standard deviation $\Delta t^{*}$ (lower figure) as a function of the current $I$ (in milliampers). Theoretical results (solid line) on the basis of (15) and (16) are compared with numerical solutions of (5)-(7) (crosses) and experimental measurements (circles). To calculate the theoretical curves, we use $G=g\left(J / J_{s w}-1\right)$ with the (reduced) parameters: $g=0.880, J_{s w}=0.962, J_{i}=0.428, \Delta_{i, f}=$ $0, \beta=3.8510^{-3}$. Experiments were performed with a $200-\mathrm{kHz}$ square wave modulation signal with a fixed lower current $\left(I_{i}=0.4 \mathrm{~mA}\right)$ and a varying upper current.

Both $\left\langle t^{*}\right\rangle$ and $\Delta t^{*}$ are inversely proportional to the dichroism $G_{f}-\Delta_{f}$, diverging when $\left(G_{f}-\Delta_{f}\right) \rightarrow 0$ as the final value of the current moves closer to the switching point. The covariance $\left\langle h^{2}\right\rangle$ also varies with dichroism. Another result that obviously can only be calculated by a stochastic analysis is the expression for the standard deviation $\Delta t^{*}$ or jitter time. It is a known result [26], [27] that $\Delta t^{*}$ does not depend on the noise strength $\beta$, nor on the reference level $p_{0}$.

In order to test all the approximations made in the analytical treatment, we compare our theoretical results with numerics, obtained by integrating the full set (5)-(7) for $10^{4}$ realizations of the noise term, and calculating the average switching time and the standard deviation from the numerically obtained distribution of the switching times. This was implemented using a object-oriented framework [28], [29] designed to facilitate the collection of said statistics. The integration procedure used was a stochastic second-order predictor-corrector method, often called the Heun algorithm. A fixed timestep of $10^{-3}$ ns was used throughout. The overall agreement (see Fig. 1) between theory (line) and numerical results (crosses) is found to be very good for the standard deviation, while still good for the average switching time. It should be noted that the agreement gets even better as the spontaneous emission rate $\beta$ decreases. For a noise level of $\beta=110^{-4}$ there was no noticable discrepancy between the theoretical and numerical results [28]. The very good agreement gives further confidence in the reduction of the three nonlinear differential equations to a single one, the linearization and the stochastic analysis in the framework of the quasi-determinisitc theory. 


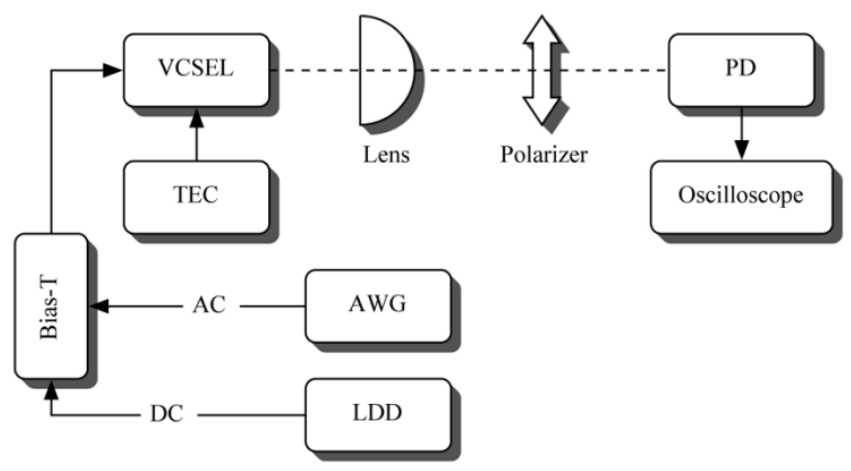

Fig. 2. Scheme of the setup used in the experiments.

\section{EXPERIMENT AND COMPARISON WITH THEORY}

The experimental results are obtained on oxide-confined VCSELs with a circular surface relief structure for better single mode operation [30]. Under the operating conditions (temperature and strain) in the experiment, this VCSEL exhibits type I PS (from higher to lower frequency with increasing current) at a current of about $0.59 \mathrm{~mA}$ (threshold $I_{\text {th }}=0.28 \mathrm{~mA}$ ) in the single fundamental mode regime. The splitting between the two polarization modes is about $13 \mathrm{GHz}$. The relaxation oscillation frequency around the PS current is of the order of $3 \mathrm{GHz}$.

In the experimental setup (see Fig. 2), the light emitted by the laser is collimated with a lens and sent through a linear polarizer to select one of the two PMs. The polarization resolved intensity impinges on 2-GHz bandwidth detector whose output signal is visualised on an oscilloscope (bandwidth $6 \mathrm{GHz}$ ). Unintentional feedback is avoided by slightly misaligning the optical components. The bias current from the laser diode driver (LDD) and the modulation signal form an arbitrary waveform generator (AWG) are combined in a bias-T and, subsequently, sent through the VCSEL. Furthermore, the temperature of the VCSEL package is actively stabilized with a thermoelectric cooler (TEC) up to a few milliKelvin. The measurements are performed by applying a $200-\mathrm{kHz}$ square wave modulation across the PS point. The standard deviation (jitter) and the rise time of the electrical input signal from the AWG were measured to be $300 \mathrm{ps}$ and $1 \mathrm{~ns}$, respectively.

A typical measured histogram of the switching time distribution is shown in Fig. 3 (upper figure). For comparison, a numerical histogram is also shown (lower figure). To calculate the average and the standard deviation from the measured distributions, the delay and finite rise time of the applied electrical signal were corected for. The so-obtained averages and standard deviations are shown by the circles in Fig. 1 as a function of the (final) value of the input current. Let us first discuss the results for the variance (lower curve), as theory predicts that it only depends on the dichroism. We suppose that the linear dichroism $G$ is linear with current I (in milliampers) for the investigated region close to the switching point where $G$ goes through zero, so (16) is equivalent to

$$
\Delta t^{*}=\sqrt{\left\langle(\Delta t)^{2}\right\rangle}=\frac{\sqrt{1.64}}{a\left(I-I_{\mathrm{up}}\right)} .
$$

By fitting the theoretical curve to the experimental data, we obtain $a=3.1(3) \mathrm{GHz} / \mathrm{mA}$ and $I_{\mathrm{up}}=0.55(1) \mathrm{mA}$. This fitted value of the switching current corresponds rather well with the approximate value deduced from the measurement of
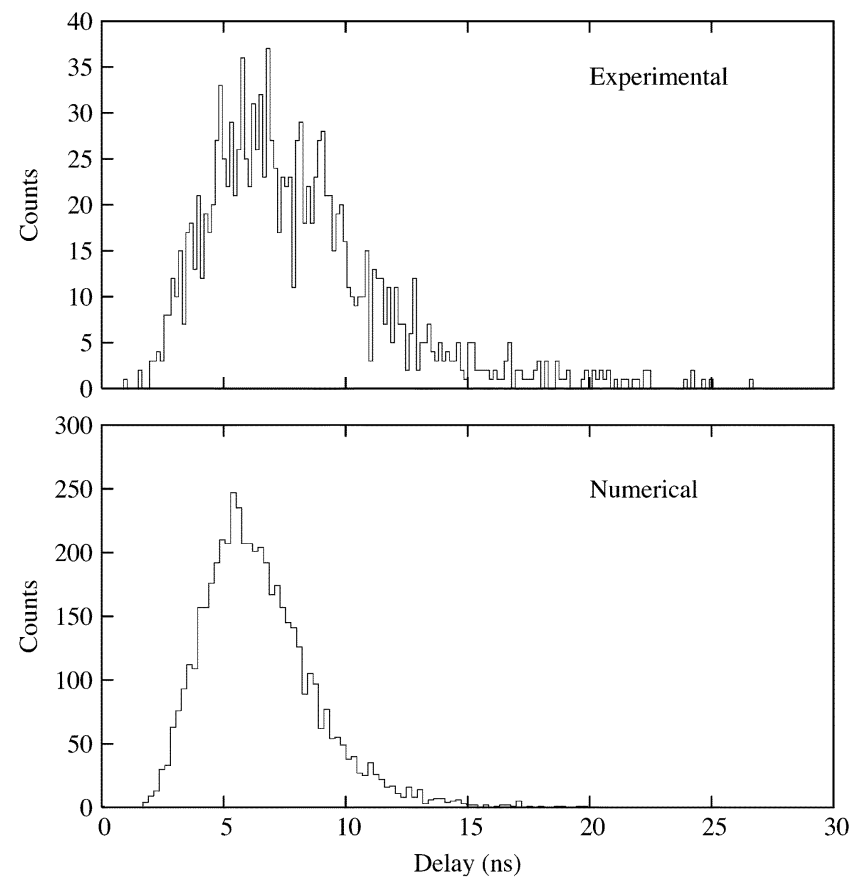

Fig. 3. Measured (upper figure) and numerically computed (lower figure) histogram of the distribution of the switching times, for a value of the final current of $0.734 \mathrm{~mA}$. From these histograms we calculate the averages and standard deviations that are depicted in Fig. 1.

the steady-state polarization resolved PI curve. The value of $a$ gives the slope of the effective dichroism (both linear and nonlinear) with the current, close to the PS current. Different physical mechanisms can contribute to $a$ : spatial hole burning, spin flip mechanisms, carrier heating, etc.

Now that the variance is fitted, we turn to the values of the mean switching time. We expect that the measured values of the switching times are less accurate than the ones of the variances, as systematic measurement errors cancel out in the variances. We keep the values of the two parameters estimated from the variances fixed, and only fit the value of the noise strength $\beta$ in (15). So doing, a value of $\beta=3.8(3) 10^{-3}$ can be deduced. One can see (Fig. 1) that the theoretical curves and the experimental data agree well. Again, the agreement is better for the standard deviation than for the average switching time.

We have also performed measurements on a similar device of the same manufacturer, but emitting at $970 \mathrm{~nm}$ and with a significantly larger bistable region around the PS current. The agreement between theory and experiment in that case is qualitatively the same as the one presented here. As for the fitted parameters, the value of the spontaneous emission rate in that VCSEL is the same as the one found here (within the experimental error), while the value for the dichroism is higher: $a=18 \mathrm{GHz} / \mathrm{mA}$. More details on these measurements will be reported elsewhere [31].

An alternative view of both the experimental and theoretical results is presented in Fig. 4, allowing to check the validity of the fittting and of the approximations made. We have plotted the inverse of the average delay and the standard deviation as a function of the current, as (15) and (16) predict that the relationships should be linear for the standard deviation, and nearly linear for the average (supposing that the dichroism is linear with current around the switching point). First of all, we note that the theoretical curve and the numerical results coincide perfectly for the standard deviation. The small deviation between the numerical 

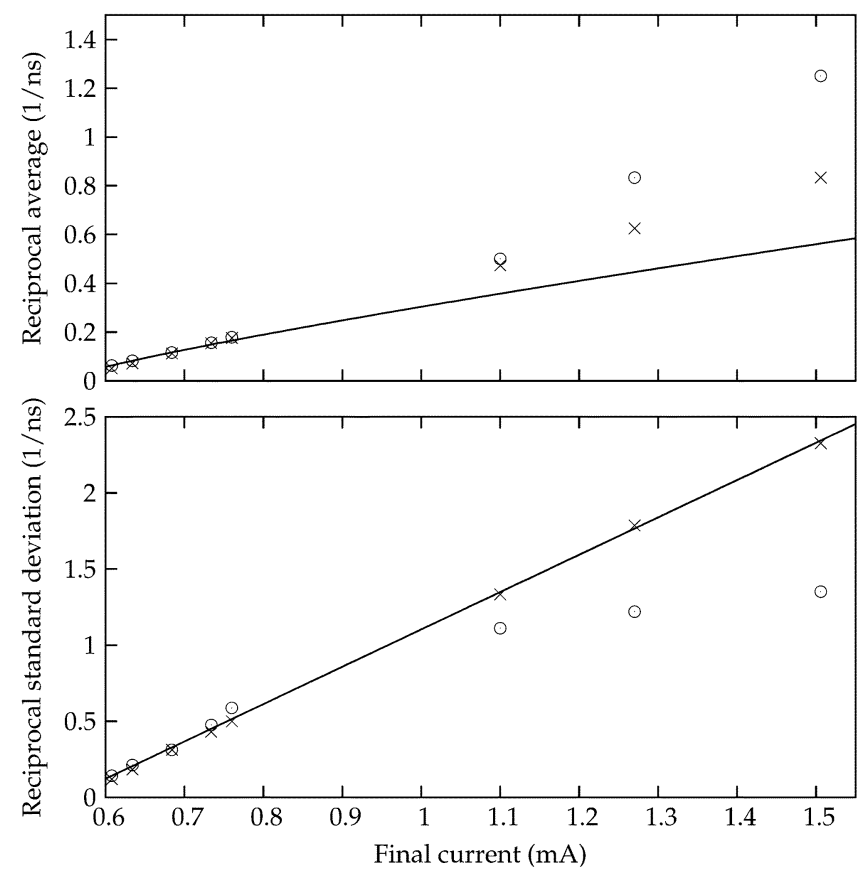

Fig. 4. Inverse of the average switching time and standard deviation versus the current. Experimental data are shown by circles, numerical results by crosses and theory by the solid curve.

simulations and theory for the average is due to the rather high noise value. Indeed, for a lower noise value $\beta=110^{-4}$, the theory is in nearly pefect agreement with simulations [28] (not shown). Fig. 4 also shows a discrepancy between the experimental points and the theoretical curve for larger values of the current step. However, these deviations are at the limit of our experimental accuracy, given by the 300-ps jitter of our measurement system. However, we cannot exclude that further physical mechanisms play a role at this level.

All theoretical and numerical results, as well as the measurements presented so far are done in the time domain. It is possible to deduce a frequency response of the polarization switching from our data: knowing the average and the standard deviation, we define a time $\tau^{*}=t^{*}+3 \Delta t^{*}$. Without going into detail about the statistical properties of the distribution, we know that a high percentage of the switches will happen within this confidence interval (if the switching times would have a normal distribution, the confidence percentage would be $99.5 \%$ ). It is then possible to define an effective frequency $f^{*}=1 / \tau^{*}$ corresponding to a modulation which the system should be able to follow (assuming the statistical properties of the down-switch are similar). In Fig. 5, we have plotted this frequency and the reciprocal amplitude of the current step, in a similar vein as was done in [9]. So, here we now derive the PS frequency response from the step response in time domain, whereas in [9], the frequency response was measured directly for other types of devices and for a lower frequency range.

As the shape of the response curve suggested a first order response, we have fitted the experimental data with

$$
H(f)=\frac{A}{\sqrt{1+\left(\frac{f}{f_{0}}\right)^{2}}}
$$

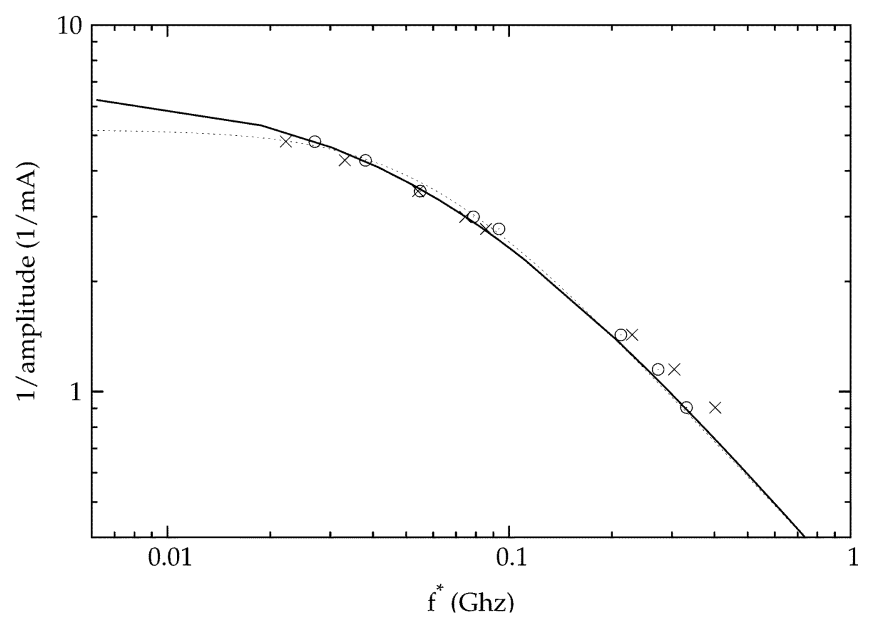

Fig. 5. Plot of the frequency $f^{*}$ at which $99.5 \%$ of the switches should be successful for a certain amplitude. Experimental data are shown by circles, numerical results by crosses, and theory by the solid curve. The dotted curve is the response of a first order system given by (18), with $f_{0}=57 \mathrm{MHz}$.

and found a good fit when the cutoff frequency $f_{0}$ is about 57 MHz. This cutoff frequency is in good agreement with a numerical limit observed in simulations of weakly index-guiding, birefringent VCSELs including spatial hole burning effects [32]; however, we want to stress that in the theory presented here no specific timescale of the gain has been taken into account, henceforth, the calculated polarization response is not limited by the material gain response time. It is instead the small relative gain/loss difference and the noise level that impose this limit.

\section{CONCLUSION}

We propose an analytical model to calculate the switching time as well as its variance as stochastic effects are clearly important in current driven PS in VCSELs. The theory is based on a rate equation approach where the clamping of the carriers allows reducing the equations to one single class-A type laser equation. The reduction of PS in VCSELs to a class-A laser gain switching problem is valid on a time scale slower than the relaxation oscillations time period, and is possible due to the clamping of the carriers above laser threshold. We can then apply the well-known theory for the statistics of a class-A laser switch-on, yielding analytical expressions for the average switching time and the jitter. The analytical approach is verified numerically, with very good agreement although small discrepancies can be noted in the average switching time for higher values of the spontaneous emission rate. Also, measurements on oxide-confined VCSELs agree well with theory. So we can conclude that PS in these VCSELs is indeed induced by the dichroism between the PMs that varies with current. As to the origin of this current-dependent dichroism, we can state that it is definitely not thermal as was the case in other VCSELs [16]. In the thermal case, the dependence of the switching times and their variance on current would be very different from the measured ones. Spatial hole burning [13] and carrier heating [14] are possible mechanisms that are compatible with the experiments reported here. In the frequency domain, the polarization modulation frequency response obtained from our measurements is 
seen to be well fitted by a first-order response with a cutoff frequency of $57 \mathrm{MHz}$. This relatively low cutoff is imposed by the small relative gain difference between the two polarization modes. From our study, this is the main factor limiting the speed of current driven polarization switching in VCSELs.

\section{ACKNOWLEDGMENT}

The authors would like to thank Dr. S. Balle (IMEDEA, Palma de Mallorca, Spain) for stimulating the present work.

\section{REFERENCES}

[1] K. D. Choquette, K. L. Lear, R. E. Leibenguth, and M. T. Asom, "Polarization modulation of cruciform vertical-cavity laserdiodes," Appl. Phys. Lett., vol. 64, pp. 2767-2769, 1994.

[2] J. Martin-Regalado, J. L. A. Chilla, J. J. Rocca, and P. Brusenbach, "Polarization switching in vertical-cavity surface emitting lasers observed at constant active region temperature," Appl. Phys. Lett., vol. 70, pp. 3350-3352, 1997.

[3] M. P. van Exter, A. Al-Remawi, and J. P. Woerdman, "Polarization fluctuations demonstrate nonlinear anisotropy of a vertical-cavity semiconductor laser," Phys. Rev. Lett., vol. 80, pp. 4875-4878, 1998.

[4] B. Ryvkin, K. Panajotov, A. Georgievski, J. Danckaert, M. Peeters, G. Verschaffelt, H. Thienpont, and I. Veretennicoff, "Effect of photon-energy-dependent loss and gain mechanisms on polarization switching in vertical-cavity surface-emitting lasers," J. Opt. Soc. Amer. B, Opt. Phys., vol. 16, pp. 2106-2113, 1999.

[5] N. Nieuborg, K. Panajotov, A. Goulet, I. Veretennicoff, and H. Thienpont, "Data transparent reconfigurable optical interconnections based on polarization-switching VCSEL's and polarization-selective diffractive optical elements," IEEE Photon. Technol. Lett., vol. 10, pp. 973-975, July 1998.

[6] S. Barbay, G. Giacomelli, and F. Marin, "Noise-assisted binary information transmission in vertical cavity surface emitting lasers," Opt. Lett., vol. 25, pp. 1095-1097, 2000.

[7] M. B. Willemsen, M. U. F. Khalid, M. P. van Exter, and J. P. Woerdman, "Polarization switching of a vertical-cavity semiconductor laser as a kramers hopping problem," Phys. Rev. Lett., vol. 82, pp. 4815-4818, 1999.

[8] B. Nagler, M. Peeters, J. Albert, G. Verschaffelt, K. Panajotov, I. Veretennicoff, H. Thienpont, J. Danckaert, S. Barbay, G. Giacomelli, and F. Marin, "Polarization-mode hopping in single-mode vertical-cavity surface-emitting lasers: Theory and experiment," Phys. Rev. $A$, vol. 68 , no. 013813 , pp. 1-8, 2003.

[9] G. Verschaffelt, J. Albert, B. Nagler, M. Peeters, J. Danckaert, S. Barbay, G. Giacomelli, and F. Marin, "Frequency response of polarization switching in vertical-cavity surface-emitting lasers," IEEE $J$. Quantum Electron., vol. 39, pp. 1177-1186, Oct. 2003.

[10] K. Panajotov, B. Ryvkin, J. Danckaert, M. Peeters, H. Thienpont, and I. Veretennicoff, "Polarization switching in VCSEL's due to thermal lensing," IEEE Photon. Technol. Lett., vol. 10, pp. 6-8, Jan. 1998.

[11] S. Balle, E. Tolkachova, M. San Miguel, J. R. Tredicce, J. Martin-Regalado, and A. Gahl, "Mechanisms of polarization switching in single-transverse-mode vertical-cavity surface-emitting lasers: Thermal shift and nonlinear semiconductor dynamics," Opt. Lett., vol. 24, pp. $1121-1123,1999$.

[12] T. Ackemann and M. Sondermann, "Characteristics of polarization switching from the low to the high frequency mode in vertical-cavity surface-emitting lasers," Appl. Phys. Lett., vol. 78, pp. 3574-3576, 2001.

[13] A. Valle, L. Pesquera, and K. A. Shore, "Polarization behavior of birefringent multitransverse mode vertical-cavity surface-emitting lasers," IEEE Photon. Technol. Lett., vol. 9, pp. 557-559, 1997.

[14] B. S. Ryvkin and A. M. Georgievskii, "Polarization selection in VCSEL's due to current carrier heating," Semiconductors, vol. 33, pp. 813-819, 1999

[15] M. Sondermann, M. Weinkath, and T. Ackemann, "Polarization switching to the gain disfavored mode in vertical-cavity surface-emitting lasers," IEEE J. Quantum Electron., vol. 40, pp. 97-104, 2004.
[16] G. Verschaffelt, J. Albert, I. Veretennicoff, J. Danckaert, S. Barbay, G. Giacomelli, and F. Marin, "Frequency response of current-driven polarization modulation in vertical-cavity surface-emitting lasers," Appl. Phys. Lett., vol. 80, pp. 2248-2250, 2002.

[17] M. San Miguel, Q. Feng, and J. V. Moloney, "Light-polarization dynamics in surface-emitting semiconductorlasers," Phys. Rev. A, vol. 52, pp. 1728-1739, 1995.

[18] G. Van der Sande, J. Danckaert, I. Veretennicoff, and T. Erneux, "Rate equations for vertical-cavity surface-emitting lasers," Phys. Rev. A, vol. 67, 2003.

[19] F. C. F. Prati and P. Caccia, "Effects of gain saturation on polarization switching in vertical-cavity surface-emitting lasers," Phys. Rev. A, vol. 66, p. $063811,2002$.

[20] B. Ryvkin, E. Avrutin, and M. Pessa, "Polarization-dependent intersubband absorption saturation and its effect on polarization on polarization selection in vertical cavity surface-emitting lasers," J. Appl. Phys., vol. 93, no. 5, pp. 2353-2358, Mar. 2003.

[21] J. Danckaert, B. Nagler, J. Albert, K. Panajotov, I. Veretennicoff, and T. Erneux, "Minimal rate equations describing polarization switching in vertical-cavity surface-emitting lasers," Opt. Commun., vol. 201, pp. 129-137, 2002.

[22] G. Giacomelli, F. Marin, M. Gabrysch, K. Gulden, and M. Moser, "Polarization competition and noise properties of VCELs," Opt. Commun., vol. 146, pp. 136-140, 1998.

[23] M. B. Willemsen, M. P. van Exter, and J. P. Woerdman, "Correlated fluctuations in the polarization modes of a vertical-cavity semiconductor laser," Phys. Rev. A, vol. 60, pp. 4105-4113, 1999.

[24] J. L. Vey, C. Degen, K. Auen, and W. Elsasser, "Quantum noise and polarization properties of vertical-cavity surface-emitting lasers," Phys. Rev. A, vol. 60, pp. 3284-3295, 1999.

[25] M. Sondermann, M. Weinkath, T. Ackemann, J. Mulet, and S. Balle, "Two-frequency emission and polarization dynamics at lasing threshold in vertical-cavity surface-emitting lasers," Phys. Rev. A, vol. 68, p. $033822,2003$.

[26] F. D. Pasquale, J. M. Sancho, M. S. Miguel, and P. Tartaglia, "Theory for the transient statistics of a dye laser," Phys. Rev. Lett., vol. 56, pp. 2473-2476, 1986

[27] M. C. Torrent and M. San Miguel, "Stochastic-dynamics characterization of delayed laser threshold instability with swept control parameter," Phys. Rev. A, vol. 38, no. 1, pp. 245-251, 1988.

[28] M. Peeters, "Polarization switching in vertical-cavity surface-emitting lasers: A modeling perspective," Ph.D. dissertation, Vrije Univ. Brussel, Brussels, Belgium, 2003.

[29] (2000-2003) Model: A modest ordinary differential equation library. [Online]. Available: http://model.sourceforge.net

[30] H. Unold, S. Mahmoud, R. Jaeger, M. Grabherr, R. Michalzik, and K. Ebeling, "Large-area single-mode VCSELs and the self-aligned surface relief," IEEE J. Select. Topics Quantum Electron., vol. 7, pp. 386-392, Mar./Apr. 2001.

[31] G. Verschaffelt, M. Peeters, J. Cordova, B. Nagler, J. Albert, H. Thienpont, I. Veretennicoff, J. Danckaert, G. Giacomelli, and F. Marin, "Timescales of polarization switching in different types of vcsels," Proc. SPIE, vol. 5452, 2004, to be published.

[32] A. Valle, L. Pesquera, and K. A. Shore, "Polarization modulation dynamics of birefringent vertical cavity surface emitting lasers," Inst. Elect. Eng. Proc.-Optoelectron., vol. 145, pp. 31-36, 1998

Jan Danckaert (M'04) was born in Antwerp, Belgium, in 1964. He received the degree in physics from the University of Antwerp in 1985, and the Ph.D. degree on the subject of nonlinear optics from the Department of Applied Physics and Photonics, Vrije Universiteit Brussel (VUB), Brussels, Belgium, in 1992.

After a stay abroad in Grenoble, France in 1993, he returned to the Department of Applied Physics and Photonics, VUB, where he is currently a Postdoctoral Research Fellow of the Flemish Fund for Scientific Research (FWO) and a Senior Lecturer. From 2001 to 2002, he was a visiting scientist at Instituto Mediterraneo de Estudios Avanzados, Universiteit de les Illes Bolears (UIB), Palma De Mallorca, Spain. He is an author or coauthor of more than 25 international journal papers. His research interests include semiconductor laser dynamics in general and the polarization and noise properties of vertical-cavity surface-emitting lasers, in particular. 
Michael Peeters (M'96) was born in Antwerp, Belgium, in 1971. He received the degree in electronical engineering (with majors in applied physics and photonics), and the $\mathrm{Ph} . \mathrm{D}$. degree on the subject of polarization switching in vertical-cavity surface-emitting lasers, from the Vrije Universiteit Brussel, Brussels, Belgium, in 1995 and 2003, respectively.

$\mathrm{He}$ is active in the development of new teaching methods for the introductory exercise courses in quantum physics, solid state physics, and electromagnetism for engineering students.

Claudio R. Mirasso was born in Buenos Aires, Argentina, in 1960. He received the M.Sc. and Ph.D. degrees in physics from the Universidad Nacional de $\mathrm{La}$ Plata, Buenos Aires, Argentina, in 1984 and 1989, respectively.

After several Postdoctoral positions in Spain and the Netherlands, he became an Associate Professor with the Physics Department, Universiteit de les Illes Bolears (UIB), in 1996. He is currently Coordinator of the European Project, Optical Chaos Communication Using Laser-Diode Transmitters (OCCULT). He has authored or coauthored over 100 publications including about 70 journal papers. His research interests include instabilities in semiconductor lasers, synchronization, and control of chaotic semiconductor lasers, vertical-cavity semiconductor lasers, and applications of nonlinear dynamics.

Maxi San Miguel was born March 3, 1952, in Barcelona, Spain. He received the Ph.D. degree in physics from the University of Barcelona in 1978.

Currently, he is Professor of physics at the Instituto Mediterraneo de Estudios Avanzados UIB-CSIC, Palma de Mallorca, Spain. Prior to that, he held professional positions at University of Barcelona, Temple University, Philadelphia, PA, Universita di Roma La Sapienza, Rome, Italy, and the University of Arizona, Tucson. His research interests include: noise, stochastic processes, and fluctuations in nonequilibrium systems; dynamics of phase transitions; nonlinear phenomena, pattern formation and spatiotemporal complexity; dynamics of social systems; nonlinear optics, quantum optics and laser physics; and semiconductor lasers.

Guy Verschaffelt (S'99-A'00) was born in Antwerp, Belgium, in 1973. He received the degree in electrical engineering (with majors applied physics and photonics) from Vrije Universiteit Brussels (VUB), Brussels, Belgium, in 1996, and $\mathrm{Ph} . \mathrm{D}$. degree on the subject of vertical-cavity surface-emitting lasers for parallel optical interconnections from the Department of Applied Physics and Photonics, VUB, in 2000.

Currently, he is working with the Department of Applied Physics and Photonics, VUB, as a Postdoctoral Research Fellow of the Fund for Scientific Research, Belgium.

Jan Albert was born in Mechelen, Belgium, in 1976. He received the degree in electronical engineering (with a major in photonics) in 1999 from the Vrije Universiteit Brussel (VUB), Brussels, Belgium, where he is currently working toward the Ph.D. degree in the polarization properties of vertical-cavity surfaceemitting lasers.

$\mathrm{He}$ is teaching basic physics exercise courses to engineering students at VUB.
Bob Nagler received the degree in electrical engineering (with majors in applied physics and photonics) and the and the Ph.D. degree in quantum electronics with a grant from the Fund for Scientific Research, Belgium, from the Vrije Universiteit Brussel, Brussels, Belgium, in 1999 and 2003, respectively.

Presently, he is with Lawrence Livermore National Laboratories, Livermore, CA.

Heiko Unold was born in Aalen, Germany, on March 25, 1973. He received the Dipl.-Ing. degree in electrical engineering from the University of Ulm, Ulm, Germany, in 1998, and the Dr.-Ing. degree in the field of single mode VCSEL design and fabrication from the Department of Optoelectronics, University of Ulm, in 2003.

Currently, he is working on passively mode-locked VECSELs at the Swiss Federal Institute of Technology (ETH), Zurich, Switzerland.

Rainer Michalzik was born in Peine, Germany, in 1965. He received the Dipl.Ing. degree in electrical engineering from the Technical University of Braunschweig, Braunschweig, Germany, in 1989, and the Dr.-Ing. degree from the University of Ulm, Ulm, Germany, in 1996.

In 1999 and 2000, he was a Guest Scientist at Bell Laboratories-Lucent Technologies, Holmdel, NJ. He is currently heading the VCSELs and Optical Interconnects Group in the Optoelectronics Department, University of Ulm, with major research interests in vertical-cavity surface-emitting laser design, fabrication, and applications, parallel and high-speed optical communication systems, as well as conventional semiconductor lasers and optical modulators.

Dr. Michalzik was awarded with the Heinz Maier-Leibnitz Prize of the Deutsche Forschungsgemeinschaft and the Federal Ministry of Education and Research in 1999 and the Merckle Research Prize in 2003.

Giovanni Giacomelli was born in 1962 in Florence, Italy. He received the degree in physics from the University of Florence in 1989 and the specialization from the School of Optics, Florence, in 1992.

Since 1994, he has been a full-time Researcher with the Istituto Nazionale di Ottica Applicata (INOA), Florence, where he is the Principle responsible for the SPICE project. His research interests include the experimental study of instabilities in dynamical systems and the experimental and theoretical study of delayed dynamical systems.

Francesco Marin was born in Italy in 1966. He received the degree in physics from the University of Pisa, Pisa, Italy, in 1990, and the Ph.D. degree in physics from the Scuola Normale Superiore, Pisa, in 1994.

He was full-time Researcher with the Physics Department, University of Florence, Florence, Italy, from 1998 to 2002. Since 2002, he has been a Professor of quantum electronics at the same University. He published about 50 articles in the fields of atomic and molecular spectroscopy, quantum optics, spicy cooking, and laser physics. 